\title{
Improved Wójcik's Eavesdropping Attack on Ping-Pong Protocol Without Eavesdropping-Induced Channel Loss
}

\author{
Zhan-jun Zhang ${ }^{1,2,3}$, Yong $\mathrm{Li}^{1}$ and Zhong-xiao $\mathrm{Man}^{2}$ \\ ${ }^{1}$ Department of Physics, Huazhong Normal University, Wuhan 430079, China \\ ${ }^{2}$ Wuhan Institute of Physics and Mathematics, \\ Chinese Academy of Sciences, Wuhan 430071, China \\ ${ }^{3}$ School of Physics \& Material Science, Anhui University, Hefei 230039, China
}

\begin{abstract}
The eavesdropping scheme proposed by Wójcik [Phys. Rev. Lett. 90,157901(2003)] on the ping-pong protocol [Phys. Rev. Lett. 89, 187902(2002)] is improved by constituting a new set of attack operations. The improved scheme has a zero eavesdropping-induced channel loss and produces perfect anticorrelation. Therefore, the eavesdropper Eve can safely attack all the transmitted bits and the eavesdropping information gain can always exceed the legitimate user's information gain in the whole domain of the quantum channel transmission efficiency $\eta$, i.e., $[0,100 \%]$. This means that the ping-pong protocol can be completely eavesdropped in its original version. But the improvement of the ping-pong protocol security produced by Wójcik is also suitable for our eavesdropping attack.
\end{abstract}

PACS number(s): 03.67.Hk, 03.65.Ud

The private key bits can be produced between two remote parties by use of quantum key distribution (QKD). QKD is a provably secure protocol since the security of QKD is based on fundamental laws of quantum physics. Many theoretical research works [2-20] have been focused on QKD since the pioneering work of Bennett and Brassard published in 1984 [1]. These QKD protocols are non-deterministic since the sender cannot determine the bit value that receiver will finally decode. Different from this kind of non-deterministic QKDs, the deterministic secure direct communication protocol is to transmit directly the secret messages without first generating QKD to encrypt them. This makes it very useful and usually desired, especially in some urgent time. However, the deterministic secure direct communication is more demanding on the security than non-deterministic QKDs. Therefore, only recently a few of deterministic secure direct protocols are proposed [21-24]. One of them is the famous Boström-Felbinger protocol, or ping-pong protocol [22], which allows the generation of a deterministic key or even direct secret communication.

In ping-pong protocol[22,25], Bob prepares two photons in the entangled state $\left|\Psi^{+}\right\rangle=(|0\rangle|1\rangle+$ $|1\rangle|0\rangle) / \sqrt{2}$. He stores one photon (home photon) in his lab and sends Alice the other one (travel photon) via a quantum channel. After receiving the travel photon Alice randomly switches between the control mode and the message mode. In the control mode Alice measures the polarization of the travel photon first in the z-basis and then announces publicly the measurement result. After knowing Alice's announcement Bob also switches to the control mode to measure the home photon in the same basis as that Alice used. Then he compares both measurement results. They should be perfectly anticorrelated in the absence of Eve. Therefore, the appearance of identical results is 
considered to be the evidence of eavesdropping, and if it occurs the transmission is aborted. In the other case, the transmission continues. In the message mode, Alice performs the $Z_{t}^{j}(j \in\{0,1\})$ operation on the travel photon to encode $j$ and sends it back to Bob, where $Z=|0\rangle\langle 0|-| 1\rangle\langle 1|$. After receiving the travel photon Bob measures the state of both photons in the Bell basis to decode the $j=0(1)$ corresponding to the $\left|\Psi^{+}\right\rangle\left(\left|\Psi^{-}\right\rangle\right)$result.

The ping-pong protocol has been claimed to be secure and experimentally feasible[22]. However, since there is a separation of the verification procedure and the key generation in the ping-pong protocol, Wójcik has presented an undetectable eavesdropping scheme on the Boström-Felbinger protocol[25]. His eavesdropping attack produces the eavesdropping-induced channel loss, therefore, requires a lossy channel to work, otherwise the eavesdropper can be detected by observing the eavesdropping-induced channel loss. Wójcik's eavesdropping scheme produces the eavesdroppinginduced channel loss at the level of $50 \%$ and the anticorrelation of the state of the home photon (kept by Bob, the legitimate receiver of secret messages) with that of the travel photon (sent by Bob to Alice, the sender of secret messages). If the transmission efficiency $\eta$ of the quantum channel is not taken into account, the probability of the eavesdropper (i.e., Eve) being detected is zero due to the anticorrelation. However, in the case of the considerable quantum channel losses, it is possible for legitimate users to detect the eavesdropping by observing the quantum channel losses. That is, although Eve can attack all the transmitted bits and the eavesdropping-induced channel loss can be hidden in the channel losses when $\eta \leq 50 \%$, if she attacks all the transmitted bits when $\eta>50 \%$, then the eavesdropping-induced channel loss is greater than the channel losses and accordingly the legitimate users can find Eve in the line by observing the channel losses.

Since Wójcik's eavesdropping scheme produces the eavesdropping-induced channel loss at the level of $50 \%$, it is desirable to have an eavesdropping scheme on the ping-pong protocol without eavesdropping-induced channel loss. To construct his attacking protocol[25], Wójcik lets Eve use both the unitary operation and the auxiliary system. since Eve is limited only by the laws of quantum mechanics, but not at all by current technology[2], she is free to use any unitary operation. In this paper, we will improve the Wójcik's eavesdropping scheme [25] by using the same auxiliary system but different set of attack operations. Our improved eavesdropping scheme indeed produces zero eavesdropping-induced channel loss and never produces both identical results of the measurements performed by Bob and Alice in the control mode.

Obviously, Eve has no access to the home photon but can manipulate the travel photon while it goes from Bob to Alice and back from Alice to Bob. Eve uses two auxiliary spatial modes $x, y$. She prepares a photon in the state $|0\rangle$ and lets the other one be an empty mode, e.g., in the state $|\mathrm{vac}\rangle_{x}|0\rangle_{y}$. Accordingly, the state of the whole system including the entangled photon pair is

$$
\mid \text { initial }\rangle=\left|\Psi^{+}\right\rangle_{h t}|\operatorname{vac}\rangle_{x}|0\rangle_{y}
$$

which is the same as Wójcik's. When Bob sends the travel photon to Alice, Eve attacks the quantum channel by manipulating the travel photon through a unitary operation (referred as to be the $B-A$ attack hereafter) as follow,

$$
W_{t x y}=U_{t x y} V_{t x y} Q_{t x y}
$$


where

$$
\begin{aligned}
& U_{t x y}=|0\rangle\left\langle\left. 0\right|_{y} \otimes \operatorname{SWAP}_{t x}+\left(\mathcal{I}_{y}-|0\rangle\left\langle\left. 0\right|_{y}\right) \otimes \mathcal{I}_{t x},\right.\right. \\
& V_{t x y}=|1\rangle\left\langle\left. 1\right|_{y} \otimes \operatorname{SWAP}_{t x}+\left(\mathcal{I}_{y}-|1\rangle\left\langle\left. 1\right|_{y}\right) \otimes \mathcal{I}_{t x},\right.\right.
\end{aligned}
$$

$\mathcal{I}$ is an identity operator, and $Q_{t x y}$ is defined in [25] as

$$
Q_{t x y}=\mathrm{SWAP}_{t x} \mathrm{CPBS}_{t x y} H_{y}
$$

which is composed of the Hadamard gate, the SWAP gate, and the three-mode gate which is called the controlled polarizing beam splitter (CPBS) by Wójcik. When acting on the initial state, the $B-A$ attack transforms the whole system to the state $|B-A\rangle=W_{t x y} \mid$ initial $\rangle$ of the form

$$
|B-A\rangle=\frac{1}{2}|0\rangle_{h}|1\rangle_{t}\left(|v a c\rangle_{x}|0\rangle_{y}+|1\rangle_{x}|v a c\rangle_{y}\right)+\frac{1}{2}|1\rangle_{h}|0\rangle_{t}\left(|v a c\rangle_{x}|1\rangle_{y}+|0\rangle_{x}|v a c\rangle_{y}\right) .
$$

Suppose that Alice now switches to the control mode and measures the state of the mode $t$. According to equation (6), we can see that after the B-A attack Alice will detect with $100 \%$ certainty a photon whose state is perfectly anticorrelated with the state of the home photon. Therefore, the probability of eavesdropping detection based on the correlation observation equals zero. This point is completely same as that in Ref.[25]. Moreover, from equation (6) we can also see that the $B-A$ attack in the present eavesdropping scheme produces zero eavesdropping-induced channel loss. In contrast, the eavesdropping-induced channel losses in [25] arrives at 50\%. This implies that, comparing to the Wójcik's eavesdropping scheme, in the present eavesdropping scheme, the domain in which Eve can attack all the transmitted bits is enlarged to $[0,100 \%]$ from the $[0,50 \%]$ in Ref.[25]. Nonetheless, this does not certainly mean that the insecurity upper bound of transmission efficiency presented by Wójcik can be pushed up, for now we still do not know the variation of the eavesdropping (legitimate) information gain. Let us now analyze the performance of the scheme in the case of Alice operating in the message mode. After Alice performs the $Z^{j}$ operation and sends the travel photon back to Bob, Eve performs her second attack (named as the $A-B$ attack hereafter) on the travel photon. The $A-B$ attack consists of the unitary operation $W_{t x y}^{-1}$. After the $A-B$ attack, the corresponding state of the whole system is

$$
|A-B\rangle_{j}=\frac{1}{2}\left[(-1)^{j}\left(\Psi_{h t}^{+}+\Psi_{h t}^{-}\right)|j\rangle_{y}+\left(\Psi_{h t}^{+}-\Psi_{h t}^{-}\right)|0\rangle_{y}\right]|v a c\rangle_{x}
$$

which is little different from the corresponding state in [25] due to the existence of the partial phase factor $(-1)^{j}$.

The final step of the eavesdropping scheme is a measurement of polarization performed on the $y$ photon. The measurement result is denoted as $k$, while Bob's Bell-state measurement on both photons is denoted as $m=0(1)$ corresponding to the $\left|\Psi^{+}\right\rangle_{h t}\left(\left|\Psi^{-}\right\rangle_{h t}\right)$ state. Assuming that Alice sends both values of $j$ with the same probability, then the only nonzero probabilities $p_{j k m}$ of possible measurement outputs are

$$
p_{000}=1 / 2, \quad p_{100}=p_{101}=p_{110}=p_{111}=1 / 8
$$


which is the same as Wójcik's. Therefore, the quantum bit error rate induced by the eavesdropping in the present eavesdropping scheme is also at the same level of $1 / 4$ as that in Ref.[25]. And the mutual information between any two parties can be easily worked out,

$$
\begin{array}{r}
I_{A E}=I_{A B}=\frac{3}{4} \log _{2} \frac{4}{3} \approx 0.311 \\
I_{B E}=1-\frac{3}{2} \log _{2} 3+\frac{5}{8} \log _{2} 5 \approx 0.074 .
\end{array}
$$

The same as Wójcik's eavesdropping scheme, the present scheme is also not symmetric. If after the $A-B$ attack Eve performs with the probability of $1 / 2$ an additional unitary operation $S_{t y}=$ $X_{t} Z_{t} \mathrm{CNOT}_{t y} X_{t} Z_{t}$, where $X_{t}$ is an negation, then the asymmetry can be removed. If the present $S_{t y}$ is performed, then the state of the whole system evolves to

$$
|A-B\rangle_{j}^{(S)}=S_{t y}|A-B\rangle_{j}=\frac{1}{2}\left[\left(\Psi_{h t}^{+}+\Psi_{h t}^{-}\right)|j\rangle_{y}+(-1)^{j}\left(\Psi_{h t}^{+}-\Psi_{h t}^{-}\right)|1\rangle_{y}\right]|v a c\rangle_{x} .
$$

Note that the present operation $S_{t y}$ is different from that in [25]. According to the viewpoint in Ref.[25], that is, since Eve knows exactly when each of the $S_{t y}$ operations has been performed, the symmetrization procedure does not reduce the mutual information between Alice and Eve while it disturbs the communication between Alice and Eve in such a way the mutual information between Alice and Bob is reduced. In the present scheme after the symmetrization procedure the mutual information between Alice and Bob is also reduced to $I_{A B}=\frac{3}{4} \log _{2} 3-1 \approx 0.189$.

Thus far, we have improved Wójcik's eavesdropping scheme by constructing a new set of attack operations. We can see that the improved scheme is almost the same as Wójcik's eavesdropping scheme except for the eavesdropping-induced channel loss. Hence, all the discussions in Ref.[25] except for those related to the eavesdropping-induced channel loss are also suitable for the present paper and we will not repeat them. Now let us discuss a very important property related to the eavesdropping-induced channel loss. In the improved scheme the $\eta$ domain in which Eve can attack all the transmitted bits is enlarged to $[0,100 \%]$ from $[0,50 \%]$ in Ref.[25]. In the $\eta$ domain of $(50 \%$, $100 \%]$, the eavesdropping information gain does not decrease in the improved scheme but it does decrease in Wójcik's eavesdropping scheme. Thus, in this sense, we can say that the present zero eavesdropping-induced channel loss does induce more eavesdropping information gain. Hence, the ping-pong protocol in its original version is insecure and can be completely eavesdropped, even in an ideal channel. Although in [22] the security proof against eavesdropping is provided, we think, it is essentially not a general proof but only the result of a special choice of the ancilla. Our present eavesdropping scheme employing two ancillas from Wójcik instead of one ancilla in [22] has shown the insecurity of ping-pong protocol.

In summary, we have improved Wójcik's eavesdropping scheme on the Boström-Felbinger quantum communication protocol. The improved scheme does not produce any eavesdropping-induced channel loss and accordingly in the whole $\eta$ domain of $[0,100 \%]$ Eve can attack all the transmitted bits. Hence, in the $\eta$ domain of $(50 \%, 100 \%]$, the zero eavesdropping-induced channel loss does induce more eavesdropping information gain. Moreover, as for as the original ping-pong protocol is concerned, it is insecure and can be completely eavesdropped, even in an ideal channel, for the eavesdropping information gain can always exceed the legitimate user's information gain. As mentioned by Wójcik[25], the ping-pong protocol can be made secure against his attack scheme 
by a modification. His improvement of the ping-pong protocol security is also suitable for our eavesdropping attack. Actually there is a significant chance of finding an additional photon in the travel mode in our attack, which can then be used as an indicator for Eve's presence. The present eavesdropping scheme introduces a qubit error rate at the same level of $25 \%$ as one for Wójcik's attack scheme. If the channel has itself a QBER significantly lower than 25\%, and if Alice and Bob sacrifice some of their message bits to perform message authentification, the qubit error rate induced by the attack can be used to detect the eavesdropper. Furthermore, another improvement of the ping-pong protocol is that, if both Alice and Bob use the generally used two-measuring-basis method [23] in the control mode, our present eavesdropping attack can also be detected with $25 \%$ possibility.

We thank to Prof. Baiwen Li for his encouragement. This work is funded by the National Science Foundation of China under No.10304022.

[1] C. H. Bennett and G. Brassard, in Proceedings of the IEEE International Conference on Computers, Systems and Signal Processings, Bangalore, India (IEEE, New York, 1984), p175.

[2] N. Gisin, G. Ribordy, W. Tittel, and H. Zbinden, Rev. Mod. Phys. 74,145 (2002).

[3] A. K. Ekert, Phys. Rev. Lett. 67, 661 (1991).

[4] C. H. Bennett, Phys. Rev. Lett. 68, 3121 (1992).

[5] C. H. Bennett, G. Brassard, and N.D. Mermin, Phys. Rev. Lett. 68, 557(1992).

[6] L. Goldenberg and L. Vaidman, Phys. Rev. Lett. 75, 1239 (1995).

[7] B. Huttner, N. Imoto, N. Gisin, and T. Mor, Phys. Rev. A 51, 1863 (1995).

[8] M. Koashi and N. Imoto, Phys. Rev. Lett. 79, 2383 (1997).

[9] W. Y. Hwang, I. G. Koh, and Y. D. Han, Phys. Lett. A 244, 489 (1998).

[10] P. Xue, C. F. Li, and G. C. Guo, Phys. Rev. A 65, 022317 (2002).

[11] S. J. D. Phoenix, S. M. Barnett, P. D. Townsend, and K. J. Blow, J. Mod. Opt. 42, 1155 (1995).

[12] H. Bechmann-Pasquinucci and N. Gisin, Phys. Rev. A 59, 4238 (1999).

[13] A. Cabello, Phys. Rev. A 61,052312 (2000); 64, 024301 (2001).

[14] A. Cabello, Phys. Rev. Lett. 85, 5635 (2000).

[15] G. P. Guo, C. F. Li, B. S. Shi, J. Li, and G. C. Guo, Phys. Rev. A 64, 042301 (2001).

[16] G. L. Long and X. S. Liu, Phys. Rev. A 65, 032302 (2002).

[17] F. G. Deng and G. L. Long, Phys. Rev. A 68, 042315 (2003).

[18]J. W. Lee, E. K. Lee, Y. W. Chung, H. W. Lee, and J. Kim, Phys. Rev. A 68, 012324 (2003).

[19] Daegene Song, Phys. Rev. A 69, 034301(2004).

[20] X. B. Wang, Phys. Rev. Lett. 92, 077902 (2004).

[21] A. Beige, B. G. Englert, C. Kurtsiefer, and H.Weinfurter, Acta Phys. Pol. A 101, 357 (2002).

[22] Kim Bostrom and Timo Felbinger, Phys. Rev. Lett. 89, 187902 (2002).

[23] F. G. Deng, G. L. Long, and X. S. Liu, Phys. Rev. A 68, 042317 (2003).

[24]F. G. Deng and G. L. Long, Phys. Rev. A 69, 052319 (2004).

[25] A. Wojcik, Phys. Rev. Lett. 90, 157901 (2003). 